\title{
Bacteriophage therapy for inhibition of multi drug-resistant uropathogenic bacteria: a narrative review
}

\author{
Zahra Chegini ${ }^{1}$, Amin Khoshbayan ${ }^{1}$, Soheil Vesal ${ }^{2}$, Alireza Moradabadi ${ }^{3}$, Ali Hashemi ${ }^{4}$ and Aref Shariati ${ }^{*}$ (1)
}

\begin{abstract}
Multi-Drug Resistant (MDR) uropathogenic bacteria have increased in number in recent years and the development of new treatment options for the corresponding infections has become a major challenge in the field of medicine. In this respect, recent studies have proposed bacteriophage (phage) therapy as a potential alternative against MDR Urinary Tract Infections (UTI) because the resistance mechanism of phages differs from that of antibiotics and few side effects have been reported for them. Escherichia coli, Klebsiella pneumoniae, and Proteus mirabilis are the most common uropathogenic bacteria against which phage therapy has been used. Phages, in addition to lysing bacterial pathogens, can prevent the formation of biofilms. Besides, by inducing or producing polysaccharide depolymerase, phages can easily penetrate into deeper layers of the biofilm and degrade it. Notably, phage therapy has shown good results in inhibiting multiple-species biofilm and this may be an efficient weapon against catheter-associated UTI. However, the narrow range of hosts limits the use of phage therapy. Therefore, the use of phage cocktail and combination therapy can form a highly attractive strategy. However, despite the positive use of these treatments, various studies have reported phage-resistant strains, indicating that phage-host interactions are more complicated and need further research. Furthermore, these investigations are limited and further clinical trials are required to make this treatment widely available for human use. This review highlights phage therapy in the context of treating UTIs and the specific considerations for this application.
\end{abstract}

Keywords: Phage therapy, Urine, Multi-drug resistant, Urinary tract infection

\section{Introduction}

Urinary Tract Infections (UTIs) constitute one of the most important concerns among medical experts and patients that are linked to almost $40 \%$ of the cases of nosocomial infections in acute care hospitals $[1,2]$. Notably, these infections are reported as one of the most common bacterial infections that affecting about 150 million people every year worldwide. UTI is one of the most common reason of morbidity and mortality in

\footnotetext{
*Correspondence: arefshariati0111@sbmu.ac.ir

1 Department of Microbiology, School of Medicine, Iran University of Medical Sciences, Tehran, Iran

Full list of author information is available at the end of the article
}

the elderly, accounting for 15.5 and $6.2 \%$ of hospitalization and deaths of people aged 65 years or older, respectively [3-5]. Women are more susceptible to UTIs than men due to behavioral factors, their anatomy, and practice such as use of diaphragm and spermicides [6]. UTIs may affect women at any age, especially women with frequent sexual activities and women of childbearing age as well as old men and infant boys [2]. UTIs are classified as either lower (confined to the bladder) or upper (pyelonephritis) and they can be clinically divided into two forms: uncomplicated and complicated. Uncomplicated form represents the conditions when patients exhibit a healthy status previous to the infection without non-catheterized, non-pregnant, and no structural abnormalities. On 
the other hand, UTI is reported as complicated when patients are immunocompromised or experience risk factors such as pregnancy and urinary retention [1, 7]. Escherichia coli is the most common (80\%) cause of the uncomplicated UTI and catheter-associated infections [8]. Other common bacteria in UTIs include Proteus species, Klebsiella pneumoniae, Staphylococcus aureus, Staphylococcus saprophyticus, and Enterococci and Enterobacter [2, 9]. Pseudomonas aeruginosa and Acinetobacter baumannii are rarely isolated from UTIs, but the epidemic potential of Multi-Drug Resistant (MDR) pathogens and high ability to cause septicemia makes these bacteria quite important [10]. Prophylaxis of UTIs or extensive and unlimited use of routine antibiotics leads to the emergence of MDR bacteria. Therefore, treating UTIs due to antibiotic resistance is becoming more difficult every day [5].

One of the main features of uropathogenic bacteria that makes them more resistant to the host immune system and chemical antibiotics is their capacity to form single- or mixed-species biofilm. This structure settles on both abiotic and biological surfaces such as indwelling urinary catheters and is characterized by low susceptibility to antibiotics and hard to remove even if the catheter is detached $[1,11]$. Thus, the high prevalence of MDR bacteria has severely restricted the use of antibiotics in recent years. Furthermore, a long course of antibiotic therapy often causes toxicity in the patients, arising antibiotic resistance and affected natural microbiota. So, interest in other treatments like phage therapy is increasing. In this context, given the phages are self-replicating, they cannot infect eukaryotic cells and their mechanisms of resistance are different from those of antibiotics $[12,13]$. Various studies suggests phage therapy has the potential to be used as either an alternative or a supplement to antibiotic treatments [14, 15]. Bacteriophage depolymerase plays an important role in the degradation of biofilm Extracellular Polymeric Substances (EPS) substrate, promoting phage penetration into the biofilm and leading to bacterial cell lysis. Indeed, this enzyme is expressed on the surface of phage capsids or produced by host cells during phage replication and it helps phages to adsorb, attack, and decompose bacterial host [16]. Furthermore, phages at the end of the lytic cycle produce endolysins. Endolysins are phage enzymes that cleave peptidoglycans, i.e., the main component of the bacterial cell wall, and they are antibacterial agents owing to their special mode of action and highly particular activities against bacteria [17]. Noteworthy, phage therapy can be divided into two categories: monophage and polyphage therapy. Two or more phages mix and they cover various bacterial hosts in a single product as a phage cocktail which is typically more effective in interfering in bacterial infections $[18,19]$.

Furthermore, recent studies have reported that despite the presence of the different factors such as biofilms and other antibiotic resistance factors in UTIs, phages along with other antimicrobial strategies may be effective in preventing and treating these infections by increasing the synergistic effect $[20,21]$. In this respect, the present review paper is an attempt at investigating more comprehensively the effectiveness of phage therapy in treating UTIs caused by MDR-uropathogenic bacteria.

\section{Escherichia coli}

Escherichia coli is one of the most common human pathogens and induces infection in different organs of the body including enteritis, septicemia, neonatal meningitis, and UTI $[22,23]$. The most frequent extra-intestinal E. coli infection is UTI and by far, the most common pathogen that induces UTI is Uropathogenic E. coli (UPEC) [24]. Moreover, the growing prevalence of MDR UPEC strains has complicated UTIs treatment and led to rise of costs and extended hospital stays [25]. Nowadays, phage therapy is considered a potential choice for treating the resistance of UPEC [13]. In this context, VB_ecoS-Golestan is reported as an alternative treatment for inhibiting UPEC. Among investigated isolates, $78.8 \%$ were MDR, of which $56 \%$ exhibited sensitivity to the lytic activity of this phage. This phage is characterized by broad host range specificity in MDR isolates; besides, none of the lysogenic mediated genes was found in this phage genome. Therefore, the authors suggested that phage therapy could be a promising approach to the treatment of MDR E. coli. However, high specificity towards $E$. coli may be a restricting factor in applying the phage to treatment of multiple-species UTI [26].

Involvement of biofilms in most chronic infections is justified by their resistance to both antibiotics and host defenses. The need for effective treatments with an ability to penetrate the biofilm structure and destroy it can bring phages into attention as an antimicrobial agent to control and prevent biofilm formation [11]. Gu et al. characterized vB_EcoP-EG1(T7-like Podoviridae family) by large burst size, no toxic protein, very short lytic cycle, and broader host range on UPEC strains. This phage brings about promising outcome as it can infect 10 out 21 MDR UPEC. In addition, this phage successfully lyses the MDR UPEC and reduces the biofilm biomass in sensitive and MDR isolates. Furthermore, vB_EcoP-EG1 shows quite a strong lytic ability in both planktonic and biofilm forms of UPEC strains. In medical settings, the biofilm induces destructive damage during acute and chronic infections such as Catheter-Associated UTI (CAUTI) by UPEC, and the phage may have a potential to treat patients with 
biofilm-related UTI [27]. In another investigation, the authors reported three phages with activity against UPEC biofilm. All phages caused a reduction in biofilm biomass compared to the untreated group at both low and higher concentrations after $8 \mathrm{~h}$ of incubation. These results demonstrate that phages act in a nondose-dependent manner, working to their advantage when applied to in vivo condition in order to remove the biofilm. On the other hand, there was evidence that biofilm start to re-stablish itself after $24 \mathrm{~h}$. This phenomenon could be linked to resistance development against the phages in bacteria [28].

Therefore, phages have a limited host range and the activation of resistance to phage in the biofilm community is one of the significant obstacles to successful phage therapy. These challenges can be overcome using phage cocktails and combination therapy of phage and antibiotics. It has already been shown that combination therapy not only reduces the number of bacteria but also relates to the management of phage-resistant bacteria levels [29]. Thus, some researches have investigated the effectiveness of combination therapy of phage and antibiotics in UPEC inhibition. In this respect, Moradpour et al. probed the synergic effect of phage and ampicillin against drug-resistant E. coli O157-associated UTI. The E. coli strain used in this study was resistant to ampicillin and had intermediate susceptibility to amoxicillin-clavulanic acid. Application of the phage resulted in keeping zones remarkably clear of ampicillin and amoxicillin-clavulanic acid in disk diffusion, reduced the overall growth of bacteria, and indicated the sensitivity of resistance phenotype in the inhibition zones. Further, the modified broth microdilution assay confirmed the phage-antibiotic synergy [30]. This synergistic effect may arise from stimulation of lytic phage growth in the presence of beta-lactams antibiotics. In fact, bacterial cells divide unsuccessfully and create very long filaments in the presence of betalactams, and this cell filamentation contributes to much faster phage assembly by simply lysing the cell. Filamentation induces perturbations in the peptidoglycan layer which may cause greater sensitivity to the activities of phage lysis [30,31]. In addition, phage attack could alter the activity of efflux pump, causing increased sensitivity to multiple classes of antibiotic agents [32]. So, findings demonstrate the significant enhancement of bacteria killing in combination therapy, compared to each treatment alone. Similarly, recent research works have been directed at effects of combined use of phages and antibiotics on E. coli strain ATCC 13,706, being resistant to different antibiotics. Phage ECA2 and antibiotics was combined and subsequently, employed to evaluate the inactivation of $E$. coli. In this regard, the combination of phage and ciprofloxacin at the sub lethal concentration remarkably reduced the bacterial level compared to using each one alone. Besides, the presence of antibiotic at a sub-lethal concentration can control the formation of phage-resistant strain. However, at sub lethal doses, antibiotics create a phage-antibiotic synergistic effect and decrease the bacterial number, but only when bacteria are sensitive to the used antibiotics. Of note, ciprofloxacin hinders the enzyme topoisomerase II (DNA gyrase) of the bacteria and, subsequently, interferes in nucleic acid synthesis, thus affecting phage replication cycle in the host bacteria. Therefore, at the Minimum Inhibitory Concentration (MIC) dose of this antibiotic, no synergistic effect could occur. Moreover, applying combination therapy in conjunction with bacteriostatic antibiotics did not lead to a synergistic effect because these antibiotics only inhibited bacterial growth and did not reduce the number of bacteria besides avoiding the phage replication. Thus, in total, the efficiency of combination therapy of phage and antibiotics is dependent on the antibiotic resistance of examined bacteria to used antibiotic and antibiotic types (bactericide or bacteriostatic) [29, 33].

As mentioned, phage therapy can be used to inhibit MDR UPEC because, besides the appropriate antibacterial effect, it can also destroy bacterial biofilms. However, rapid emergence of phage-resistant bacteria has limited their use and it is the reason why use of phage cocktails and combination treatments should be studied further.

\section{Proteus species}

Proteus species play a major role in UTIs and infections caused by these pathogens divided into two types: (a) hematogenous infections, e.g., systemic infections, and (b) ascending infections assisted by bacteria to be colonized step by step in the urinary tract and to reach the kidney eventually $[34,35]$. Warren et al. described P. mirabilis, followed by E. coli and K. pneumoniae, as the third most common pathogen associated with the complicated UTI at a prevalence rate of $12 \%$ for infections [36]. Furthermore, CAUTI occurs in $50 \%$ of all patients catheterized for 7 days or more, and $P$. mirabilis is the predominant organism in $44 \%$ of CAUTIs [37-39]. Although treatment with antibiotics is effective in managing most of cases, rise of antibiotic resistance among CAUTI-causing bacteria, including $P$. mirabilis, makes the CAUTI treatment harder. This phenomenon highlights the importance of novel methods and phage therapy has reemerged to meet medicinal objectives in the last decade [40, 41].

In this context, two novel virulent phages including vB_PmiP_5460 and 5461 are introduced which belong to Podoviridae and Myoviridae families, respectively. These phages are used as cocktail-coated catheters to evaluate the possible effect of biofilm reduction, compared with the non-phage coated catheters. A reduction in 
biofilm population in phage-coated catheters is observed. Moreover, a clear tendency of phage cocktail leads to the remarkable reduction of biofilm formation in 96 and $168 \mathrm{~h}$ after catheterization. These effects are promising since urinary catheters remained in the patients' bladder for a long course and the potency of the phage-coated catheters lasted for 7 days in this study [42]. Moreover, in another investigation, different degrees of sensitivity to 13 phages in 50 isolates of uropathogenic $P$. mirabilis in planktonic and biofilm forms were reported. Among the 13 phages, 39APmC32, 65APm2833 (Myoviridae family), and 72APm5211 (Siphoviridae family) were selected as cocktail components. These phages exhibited a strong anti-biofilm activity that had a better potential to inhibit biofilm formation rather than to remove mature biofilms [43]. It appears that phages can delay the formation of biofilms or even hinder their formation by inhibiting the growth of $P$. mirabilis (Fig. 1). Another good anti-biofilm feature of phages 39 and $72 \mathrm{~A}$ is the production of polysaccharide depolymerase, demonstrating the formation of halo around plaques. Besides, these phages eradicate more than $50 \%$ of biofilms in the investigated strains. In this way, phage-encoded polysaccharide depolymerase degrades bacterial exopolysaccharide which is the main component of biofilm matrix. It is remarkable that halos are formed by bacteria from which exopolysaccharide has been removed through excess phage enzyme released during the lysis of infected cells [44, 45]. Furthermore, applying the phage cocktails containing several phages is a reasonable solution for phages with a narrow host spectrum. In a similar fashion, in this study, phage $65 \mathrm{~A}$ has a low potential for destroying biofilms, but is characterized by the widest range of hosts. Authors recommend that the addition of the mentioned phage to the phage cocktail preparation could lead to increase in the number of bacteria susceptible to the cocktail, compared to preparations containing single phages. Thus, phages 72 and $39 \mathrm{~A}$ produce polysaccharide depolymerases that promote the penetration of phage $65 \mathrm{~A}$ into the deeper biofilm layer [43].

Similarly, Nzakizwanayo et al. reported three specific P. mirabilis phages which belonged to Podoviridae family. All of these phages exhibit polysaccharide depolymerase activity against their host. Furthermore, in the worst possible condition where phage is used to treat an established infection, use of a single-dose phage cocktail has remarkably (3-fold) prolonged the time taken for blocking catheters. However, the application of the same phage dose in the early phase of infection has led to complete inhibition of catheter blockage and eradication of infection. Additionally, phage treatment of biofilm formation caused a significant decrease in the level of encrustation, compared to control group. The remarkable point highlighted by this study is the importance of utilizing the phage in the early stage of infection, thus leading to full prevention of blockage. In fact, this phenomenon may be characterized by the insufficient dose of phage to deal with dense bacterial population or the development of resistance to the utilized phage [46, 47]. Furthermore, another research reported five phages with a remarkable ability to disrupt pre-formed MDR P. mirabilis biofilms, thus reducing the number of viable cells by $99.9 \%$ [48]. Moreover, combination therapy of ampicillin and phage vB_PmiS-TH against planktonic and biofilm forms of $P$. mirabilis recovered from UTI was used in another work. In the planktonic culture, the bacterium was highly susceptible to combination therapy. Notably, phage-antibiotic combination had the highest effect on the removal of biofilm after $24 \mathrm{~h}$, which demonstrated the superiority of combination therapy to each of therapies alone in terms of efficiency. In addition, this treatment could inhibit the development of resistance mutant that develops quickly in exposure to each one of phage or antibiotics [49].

Up to $30 \%$ of all urinary tract stones (struvite) are created by $P$. mirabilis crystalline-shaped biofilms in the urinary tracts. The flow through catheters is recurrently blocked by these crystalline structures and then, bacteria embedded in crystalline biofilms become highly resistant to immune system as well as routinely used antibiotics $[50,51]$. In this regard, finding a treatment to inhibit the biofilm of this bacterium can prevent CAUTI. As reported in the mentioned studies, phages can have excellent inhibitory performance against $P$. mirabilis biofilm, although further studies are required to determine how they can be used to maximize efficacy. In addition, the use of phage cocktails can target a wider range of microorganisms, and the use of a combination of phages with antibiotics increases the chances of eradicating the infection. Therefore, the use of phages to control CAUTI caused by Proteus species should draw much more attention.

\section{Klebsiella pneumoniae}

Klebsiella pneumoniae are reported as one of the main opportunistic pathogens with the ability to cause hospital-acquired UTIs predominantly in the elderly, newborns, and immunocompromised individuals [52]. Dissemination of antibiotic resistance, especially to carbapenem, fluoroquinolones, and colistin, is becoming increasingly serious and only a few remaining therapeutic options remain to treat Pan-Drug-Resistant (PDR) $K$. pneumoniae infections. In this respect, the spread of PDR $K$. pneumoniae species worldwide threatens modern medicine to revert to its pre-antibiotic era [53, 54]. Thus, one of the possible options to inhibit these pathogens is to use phages due to their well-defined target spectrums 
and host range specificity [55]. In this section, we will discuss the specific function of phage therapy to inhibit K. pneumoniae in UTIs.

Sybesma et al. isolated nine $K$. pneumoniae strains (includes ESBL-producing strains) from urinary culture of different patients with UTIs and investigated the in vitro susceptibility to phages. Notably, all of these lytic phages were prepared from the phage collection of the George Eliava Institute of phage and were used in the bacterial cell lysis screening assay. The authors reported that v_BR-KpS10 could lyse all $K$. pneumoniae strains. Hence, this study proposed that existing commercial phages could be used more to control MDR K. pneumoniae UTI. Furthermore, the data in this study indicated that phage and antibiotic susceptibility/resistance were not related to each other and although there was resistance to various antibiotics in the K. pneumoniae strains, they were all lysed by one of the phages [56]. In another study, one lytic phage, KPO1K2, was isolated from effluent water to inhibit $K$. pneumoniae. This phage was a member of Podoviridae family with T7-like specifications and was proved stable over a wide $\mathrm{pH}$ range of 4-11. According to the spot test, phage was found to infect $28 \%$ (7/28) of K. pneumoniae. Furthermore, phage kinetics for its in vivo stability was conducted in mice. After intraperitoneal injection into mice, KPO1K2 titers in urinary bladder and kidney clearly indicated that phage accumulation occurred at higher concentrations in these two organs. Notably, in addition to $K$. pneumoniae, KPO1K2 infected $E$. coli. Since these bacteria are known for causing UTI, interestingly, both of them are sensitive to a single phage [57]. In a similar study by Zhang et al., novel phage vB_KpnP_IME279 was isolated from hospital sewage for lysis of MDR K. pneumoniae isolated from urine. This phage belongs to Podoviridae and is stable in a wide $\mathrm{pH}$ range between 3 and 11 and temperature range between 40 and $60{ }^{\circ} \mathrm{C}$. It should be noted that vB_KpnP_IME279 has lytic functions against several tested $K$. pneumoniae. Furthermore, phage genome analysis reveals that the investigated phage does not contain a toxin gene, which provides safety guarantee for the future clinical treatment of this phage [58]. Therefore, the reported phages did not lose their activity even at extremes of $\mathrm{pH}$; thus, it appears that they enjoy higher and longer stability in urinary bladder and kidney. In this context, these studies recommended phage therapy as a suitable candidate and a biocontrol strategy to treat MDR K. pneumoniae-associated UTI; however, control trails in this field are essential.

Recent researches reported $K$. pneumoniae capsule and biofilm as the most important virulence factors that have multiple functions such as adhesion to different surfaces and protection of the bacterium from lethal serum components, immune clearance, and environmental factors [53, 59]. Therefore, isolation and identification of phages that can inhibit and destroy these bacterial virulence factors is necessary to control UTI. In a recent publication, Phage 117 and Phage 31 were isolated and characterized. Then, the host range and lytic potential of these phages were tested by spotting nine carbapenamase-producing and hypermucoviscous K. pneumoniae (ST11 and blaKPC-2 gene positive) isolates from elderly patients with UTI. The use of each phage alone provided the potential to lyse K. pneumoniae strains. Subsequent culture and urine experiments with Phage 117 showed a strong lytic activity of the phages at first. Nevertheless, observation of rapid regrowth following the initial lysis suggested that phage-resistant mutants were selected among the host populations. On the other hand, the phage cocktail $(117+31)$ had remarkably higher antimicrobial activity than Phage 117 alone. In this respect, the authors suggested that phage cocktail could act as a promising choice for phage therapy to control $K$. pneumoniae due to its potential ability to infect phage-resistant mutants and thus, delay the development of bacterial resistance. Although phage predation affected the assembly or synthesis of capsule expression, it had no significant effect on the biofilm formation [60]. Also, another study reported a phage, vB_KpnS_Kp13, that was effective against all Verona integron-encoded metallo- $\beta$-lactamase producing $K$. pneumoniae (ST15 and expressing the K24 capsule) originating from different hospital samples such as urine. Although this phage showed a narrow host range, no phage-resistant strains were identified in this study; thus, this phenomenon strongly suggested that the target of this phage could be related to strain survival. Of note, vB_KpnS_Kp13 had a quite efficient function in killing encapsulated bacteria; the results of analyzing its genome showed that the specific feature of the capsule depolymerase, encoded by ORF2, of this phage provided the mentioned function. Furthermore, the phage significantly degraded biofilm and reduced the biomass by $\sim 73 \%$ in $48 \mathrm{~h}$ post-treatment [55].

In this respect, in another study, a phage 0507-KN2-1 was isolated for a new capsular type of $K$. pneumoniae (KN2) UTI isolates. This phage was categorized as a member of the Myoviridae phage family and the analysis of the genome indicated a putative polysaccharide depolymerase encoded by 3738-bp gene. A recombinant form of this protein was produced and was analyzed for confirmation of its enzymatic activity and specificity to capsular polysaccharides. Then, in spot tests, this purified depolymerase led to decapsulation of K. pneumoniae strains. Notably, this protein was specific to KN2 capsular polysaccharides and was not able to induce digestion-like 
spots on K. pneumoniae strains with another type of capsule or KN2 capsular polysaccharide mutant [61]. Furthermore, in another work, Siphoviridae phage TSK1 showed a potent lytic activity against $K$. pneumoniae. This phage had a narrow host range and enjoyed the highest stability at $\mathrm{pH} 7$ at a temperature of $37^{\circ} \mathrm{C}$. Besides, posttreatment with TSK1 caused a reduction in K. pneumoniae biofilms biomass (of different ages). Pre-treatment of $K$. pneumoniae biofilm with TSK1 decreased biomass $>99 \%$ in the first $24 \mathrm{~h}$ of incubation. Therefore, this study recommended that due to TSK1 stability at alkaline $\mathrm{pH}$, this phage can be employed as a medicinal agent for K. pneumoniae-mediated UTIs, while it is not suitable for direct oral administration. Also, due to the higher ability of TSK1 to destroy bacterial biofilm, this phage can be utilized in the impregnation of urinary catheter to hinder CAUTIs [53].

It seems that phages and recombinant depolymerase can be used as therapeutic strategies to control K. pneumoniae UTI, given that phages can prevent or destroy the most important virulence factors of this bacteria such as capsules and biofilms through capsule-polysaccharide specific depolymerase. Of note, this enzyme is required for degradation of capsule and adsorption onto the host cell. Consequently, following the degradation of capsule, the exposure of outer membrane components to phage for better accessibility occurred. On the other hand, according to findings, phage depolymerase acts specifically against a specific capsule type and isolated phages usually have a narrow host range and can only be used for a specific bacterial strain. Therefore, phage can be employed in combination with other phages in phage cocktails and with antibiotics to combat $K$. pneumoniae in UTIs.

In general, in addition to UPEC, Proteus species, and K. pneumoniae that have been studied further in the field of phage therapy to control UTIs, other researchers have investigated the effect of phages on uropathogenic bacteria. In this manner, Table 1 lists the recent related studies that used phages to inhibit different uropathogenic bacteria.

\section{Multi-species cocktails}

The prevalence of antimicrobial resistance causes obstacles to a successful treatment and also, biofilm-based infections create even more challenges these days; therefore, phage therapy could be a useful choice in combating MDR uropathogenic bacteria such as Proteus spp. and E. coli. In this manner, a study investigates the activities of three commercial phage cocktails (Septaphage, PYO and INTESTI) against 70 MDR E. coli and 31 Proteus spp. (including 15 MDR strains) collected from human

Table 1 Recent studies used phage therapy to inhibit the most common uropathogenic bacteria

\begin{tabular}{|c|c|c|c|c|}
\hline Uropathogenic bacteria & Properties & Phage & Outcome & Reference \\
\hline Enterobacter cloacae & MDR & $\begin{array}{l}\mathrm{E}-2, \mathrm{E}-3 \text { and } \mathrm{E}-4 \text { were isolated from waste } \\
\text { water }\end{array}$ & $\begin{array}{l}\text { The growth of the bacteria was inhib- } \\
\text { ited by the three phages. Notably, } \\
\text { the use of cocktails with two or three } \\
\text { phages was significantly more effec- } \\
\text { tive than each one alone. In urine, the } \\
\text { inactivation was still effective }\end{array}$ & {$[65]$} \\
\hline Enterococcus faecalis & Clinical isolates & $\begin{array}{l}\text { VB_EfaS_GEC-EfS_3 (Siphoviridae) isolated } \\
\text { from sewage }\end{array}$ & $\begin{array}{l}\text { While phage was able to infect a broad } \\
\text { range of strains of the same species } \\
\text { as the host species from which they } \\
\text { were isolated, they were unable to } \\
\text { infect other host species tested }\end{array}$ & {$[66]$} \\
\hline Enterococcus faecalis & VRE & $\begin{array}{l}\text { vB_EfaS_HEf13 (genus Sap6virus in the } \\
\text { family Siphoviridae) was collected from } \\
\text { a local sewerage system }\end{array}$ & $\begin{array}{l}\text { The lytic activity of phage HEf13 at } \\
\text { various multiplicities of infection } \\
\text { consistently inhibited the growth of } \\
\text { diverse clinical isolates of E. faecalis } \\
\text { without any lysogenic process }\end{array}$ & {$[67]$} \\
\hline Streptococcus mitis & Clinical isolates & $\begin{array}{l}\text { vB_SmM_GEC-SmitisM_2 (Myoviridae) } \\
\text { isolated from sewage }\end{array}$ & $\begin{array}{l}\text { This phage was able to productively } \\
\text { infect } 9 \text { of } 16 \mathrm{~S} \text {. mitis strains, but none } \\
\text { of the other species in our collection }\end{array}$ & {$[66]$} \\
\hline Staphylococcus saprophyticus & MDR Clinical isolates & $\begin{array}{l}\text { vB_SsapS-104 (Siphoviridae) was isolated } \\
\text { from hospital wastewater }\end{array}$ & $\begin{array}{l}\text { This phage represented high anti- } \\
\text { bacterial activities against S. sapro- } \\
\text { phyticus isolates in vitro, as it was } \\
\text { able to lyse } 8 \text { of the } 9 \text { clinical isolates } \\
\text { (88.8\%). Notably, no lytic activity was } \\
\text { observed on some other pathogenic } \\
\text { bacteria tested }\end{array}$ & {$[68]$} \\
\hline
\end{tabular}


and non-human origins. These cocktails were produced by Georgian institutions including PYO and INTESTI from Eliava BioPreparations and Septaphage from Biochimpharm. The percentages of the susceptibility of E. coli strains to PYO, INTESTI, and Septaphage were $61.4 \%, 67.1 \%$, and $8.6 \%$, respectively. Also, the susceptibility of Proteus spp. to PYO, INTESTI, and Septaphage was $29.0 \%, 38.7 \%$, and $19.3 \%$, respectively [62]. Interestingly, Septaphage almost represented no activity against the investigated strains. This difference among biopreparations results from different production methods and contents that caused insufficient phage titer of the final product [63]. Therefore, it appears that the function of commercial phage cocktails to inhibit MDR bacteria is more limited. This narrow activity could result from the absence of specific phages targeting contemporary MDR strains which are spreading in different settings. Therefore, the specific phages for the emerging MDR bacteria should be isolated, well characterized, and then, integrated into conventional biopreparations [62, 64]. Because it is only in this case that commercial phage cocktail can be used to treat MDR UTI around the world.

\section{Phages inhibition for other uropathogenic bacterial biofilm}

As discussed in the previous sections, phages have the ability to destroy the biofilm of bacteria that cause UTI; however, during the course of this infection, different bacteria forms mixed-species biofilm on in-dwelling medical devices or host organs. So, using a specific phage of bacterial species cannot completely eradicate the infection [69].

In a recently published paper, a cocktail consisting of MDR A. baumannii infecting phages (Aba 1-6) with highly lytic activities varying from 56 to $84 \%$ was used to destroy the bacterial biofilm in combination with antibiotics in human urine. The usage of all phages significantly reduced $A$. baumannii biofilm biomass, and Aba-1 appeared to be having the highest antibiofilm activity in the urine condition. Furthermore, the use of phage cocktail in combination with trimethoprim/ sulfamethoxazole (SXT) and ciprofloxacin led to biofilm biomass reduction rates of 94.3 and $93.3 \%$, respectively. Furthermore, in most cases synergistic effect also causes the limitation of persister cell regrowth. Of note, combination therapy with gentamicin, tobramycin, imipenem, and meropenem similarly induced a more significant reduction in $A$. baumannii biofilm biomass than the phage cocktail-treated control. On the other hand, combination therapy with colistin did not present the same expected outcome as its mentioned counterparts mentioned above. The synergistic impact can be clarified by the antibiotics mode of activity. Antibiotic-related changes within the bacterial cell morphology empowered fast phage development and cell lysis. Besides, the set of phage lytic enzymes, particularly those responsible for local peptidoglycan degradation, could enhance antibiotic penetration through biofilm matrix. On the other hand, combination of phage and colistin did not have synergistic effect because colistin destabilized the bacterial cell membrane and could limit phage propagation. In addition, overexpression of the efflux pump and autoinducer synthase AbaI that accelerate the synthesis and transport of acylated homoserine lactones can be related to the increase of biofilm formation. Therefore, combined treatment can destroy the biofilm of MDR A. baumannii in UTI through such mechanisms as enzyme-mediated permeabilization of membranes, digestion of the biofilm matrix, and enhancement of antibiotics activity. In addition, phage therapy can target persister cells through antibiotic treatment. Nevertheless, this activity may depend on the type of bacterial pathogen and the antibiotic used [10,70].

In another investigation by Yang et al., PHB08, a virulent E. faecalis phage, and its endolysin (lys08) demonstrated antibiofilm activity against E. faecalis biofilms isolated from UTI. Different analyses revealed that the mentioned phage belonged to Siphoviridae family and exhibited great stability at different temperatures (4-60 ${ }^{\circ} \mathrm{C}$ ) and pHs (between 5.0 and 9.0). Furthermore, this phage infected 79\% (15/19) of the E. faecalis clinical isolates tested, but had no effect on other bacterial species such as E. faecium. Both phage and its endolysin remarkably decreased bacterial biofilm density. So, this study also suggested phage and endolysin as possible biocontrol agents that could destroy the biofilm formation of $E$. faecalis. Additionally, high stability of phages in different environmental conditions can increase their use in UTI [71]. Another interesting study on two-species biofilms investigated the effectiveness of hydrogel silicone urinary catheter treated with phage cocktail in inhibition of biofilm formation by a mixture of $P$. mirabilis and $P$. aeruginosa in an in vitro model using artificial urine. The observations indicated that pre-treatment with phage led to reduction of $P$. mirabilis biofilm number by $>2 \log _{10}$ $\mathrm{CFU} / \mathrm{cm}^{2}$, while this value was $4 \log _{10} \mathrm{CFU} / \mathrm{cm}^{2}$ for $P$. aeruginosa over $48 \mathrm{~h}$. Notably, the presence of $P$. mirabilis always causes an increase in lumen $\mathrm{pH}$ from 7.5 to 9.5 and such a high $\mathrm{pH}$ has no inhibitory effect on the function of phages in the catheter model. On the other hand, survival of bacterial population was reported for both pathogens in the anti-biofilm assay. However, plaque formation observed on the plates with the recovered adherent bacteria implied that large fractions of these bacteria were not truly phage resistant [72]. This phenomenon may be related to metabolic alternation of bacteria in 
biofilm community, especially cells in the dipper layer of biofilm that did not support phage replication following infection. Another alternative explanation could be "spatial refuges" in which resistant bacteria create a physical barrier for susceptible cells against phage invasion [44, 72]. Therefore, the authors proposed that it was possible to use phages in indwelling urinary catheters with high $\mathrm{pH}$ conditions. Additionally, phages can be used to inhibit multiple species in mixed-species biofilms without interfering in the lytic capacities of the other phages. These findings demonstrate the possibility of applying a phage cocktail to CAUTIs because the introduction of a foreign body in the urinary tract facilitates higher microbial colonization and mixed-species biofilm formation.

In another research, it was also reported that pre-treating urinary catheter with $P$. aeruginosa specific phage ФE2005-A and E. coli HU2117 had a synergistic protection against the formation of $P$. aeruginosa biofilm on catheters. In this regard, silicone catheter segments were exposed to phage plus $E$. coli and challenged by $P$. aeruginosa; then, incubation occurred in the human urine sample up to $72 \mathrm{~h}$. The result showed that this pre-treatment led to the lower adherence of $4 \log _{10}$ unit of $P$. aeruginosa to the catheter than no pre-treatment. It is worth mentioning that due to the specificity of the phage host, the presence of special Pseudomonas phage did not considerably interfere in E. coli HU2117 communities [21]. These discoveries revealed that the combination of phage and probiotic bacterium interference would lead to the attenuation of biofilm formation by $P$. aeruginosa on urinary catheters. This phenomenon results from the fact that the use of specific $P$. aeruginosa phage reduces the initial population of this bacterium, a process that allows the $E$. coli biofilm to be established easier. Therefore, mixed biofilm does not form because the combined effect of microbial competition and lytic phage infection may lead to the predominance of one species in the biofilm. However, the use of phages and $E$. coli alone did not show good outcomes Because it seems that $E$. coli was unable to outperform $P$. aeruginosa and using the phage solitarily was obviously insufficient to destroy all $P$. aeruginosa cells prior to mixed biofilm formation on the catheter [21, 73].

The definite role of catheter-associated biofilms in UTI is poorly known; however, there is evidence that this kind of biofilms has a major role as the stable reservoir of uropathogenic microorganisms, which are resistant to antimicrobial and it is difficult to eliminate them even with the catheter removal. Therefore, prevention and removal of bacterial biofilm is very important in the treatment of UTIs. In this context, phages have been reported as one of the most significant candidates for inhibiting bacterial biofilm. However, bacterial biofilms are usually produced by several different pathogens and cannot be completely eradicated using a specific phage (Fig. 1) [74]. Therefore, as mentioned in the above studies, the combined use of several different phages as phage cocktail, the combined treatment of phages and antibiotics, and the use of probiotics along with phages can help inhibit the mixed biofilm further. Furthermore, it may be useful to investigate the possible synergistic interactions between phage cocktails and other antimicrobial strategies such as biofilm inhibitors, catheters with more routine antibacterial substances or bacterial interference. Notably, despite the use of different combination therapies, phage-resistant bacteria are still reported in studies; however, with the use of combination therapies, their population is greatly reduced. Therefore, it is very important to identify more effective strategies to kill all bacteria in the biofilm and prevent the development of resistance to phages. In doing so, the true value of these treatments is determined when used in in vivo and clinical studies.

\section{In vivo and clinical use of bacteriophage for the treatment of UTIs}

As fully discussed in the previous sections, the use of phages to inhibit bacteria that cause UTIs has been reported in many in vitro studies. On the other hand, phage therapy has not been well received in clinical and in vivo researches. Nonetheless, we will discuss clinical and in vivo studies that have used phages to control and treat UTIs.

In the case of a 60-year-old male patient who underwent kidney transplantation, multiple chronic UTIs with an MDR K. pneumoniae were reported. During each hospital stay, the patient was treated for 12-14 days with meropenem alone or meropenem with colistin and it yielded rapid, yet temporary, resolution of infection symptoms. After 10 severe cases of UTI with hospitalizations, phage therapy was used to treat the infection. In this regard, the intra-rectal application $(10 \mathrm{ml}$ twice daily) of the specific phage preparation against the MDR K. pneumoniae strain was used; however, after five days, the patient's condition worsened and ultrasound examination showed polycystic kidneys. Therefore, a combination of meropenem and phage was used and after 18 days of this treatment, progressive remission of clinical signs was observed. Due to multiple recurrent infections and unsuccessful outcomes, the patient was selected eventually for a planned resection of his left polycystic kidney [75]. In this patient, the main source of infection was cysts in the left kidney that caused several therapeutic failures; surgical intervention achieved the best therapeutic outcomes. Furthermore, due to the combined use of phage and antibiotics for the treatment of the patient, it is difficult to evaluate the exact function of phage therapy, but it seems that the infection in the transplanted 


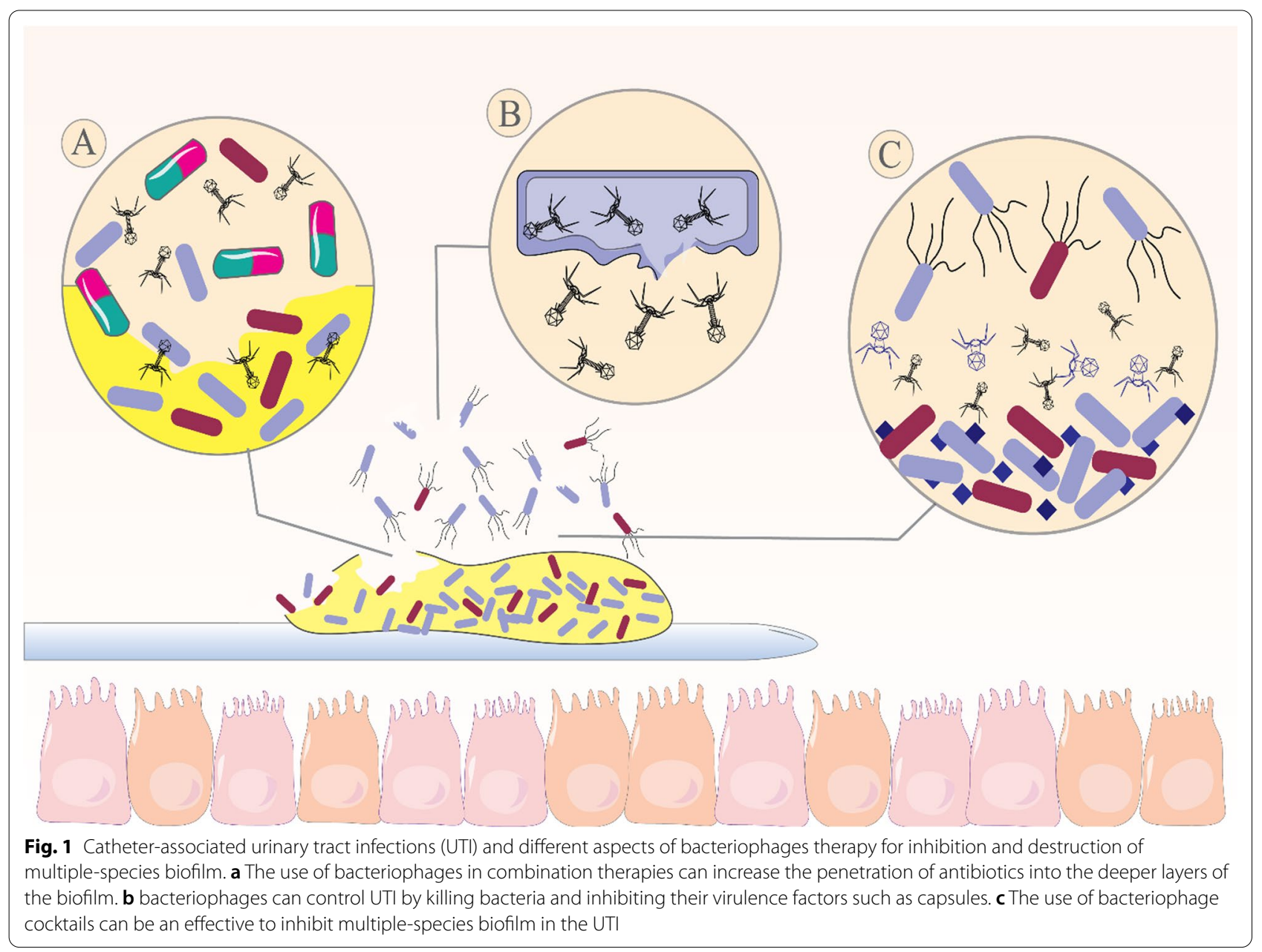

kidney could be controlled through phages. On the other hand, shortly after the use of phage therapy, it is possible that phage may have facilitated the selection of phageresistant bacteria. Thus, the use of phage cocktails or combination therapies with antibiotics can be helpful [76]. Notably, K. pneumoniae isolated from the patient were completely sensitive to the colistin; however, after 12 days, antibiotic therapy stopped on the suspicion of nephrotoxicity. However, long-term (29 days) use of phages and phage lysate application were safe, particularly in relevance to the long-term kidney function and allograft tolerance.

Phage therapy was also used in another 63-year-old female patient with recurrent UTI caused by Extensively Drug-Resistant (XDR) K. pneumoniae. It is noteworthy that, this bacterium was completely resistant to all tasted antibiotics except polymixin B and tigecycline. This patient was treated by antibiotics, but due to antibiotics utilization, complication became increasingly severe. Hence, she agreed to a phage therapy clinical trial and then, phage cocktail consisted of five lytic phages, used for the first round by bladder irrigation. This phage therapy regimen (Cocktail I) failed to completely kill the bacteria, and phage-resistant bacteria were isolated. In this respect, another cocktail (Cocktail II) was used, which included specific phages against bacteria isolated from the patient. Unfortunately, phage-resistant bacteria were isolated again. Thus, it was hypothesized that the combined use of phages and non-active antibiotics against bacteria could be helpful and in this way, the combined use of bladder-irrigated cocktails (Cocktail III) and oral SXT showed strong synergistic effect and the recurrent UTI symptoms subsequently disappeared [20]. Therefore, the findings of this study suggested combining the phage cocktail with antibiotic in the treatment of recurrent UTI in order to lower the risk of regrowth of phage-resistant mutants. It seems that the rapid onset of phage-resistant strains has been due to the poor functioning of the patient's immune system, given that a proper immune system function is essential to the clearance of bacterial infection [77]. However, more molecular studies are 
required to understand the exact mechanism of resistance to phage cocktails.

A recently published study by Ujmajuridze et al. evaluated different characteristics of phage therapy such as feasibility, tolerability, safety, and clinical/microbiological outcomes in patients with UTIs. In this way, PYO phage (phage cocktail consisting of phage lines is activated against a broad spectrum of uropathogenic bacteria) produced by Eliava was used. After in vitro phage sensitivity test, the hospital was informed of PYO phage solution to initiate the clinical analysis. After Transurethral Resection of Prostate (TURP), suprapubic and transurethral catheters were inserted to maintain phage cocktail irrigation. Then, after one to two days, the transurethral catheter was removed, while the suprapubic catheter was kept in place for another 7 days to facilitate the adapted phage cocktail instillation. Total sensitivity of $41 \%$ was reported in in vitro analysis. Besides, in in vivo pilot series, $67 \%$ (6/9) of patients' bacterial titers were reduced after phage treatment. Notably, no phage-associated side effect was reported. So, the authors suggested that phage therapy could be safe and effective in UTI treatments [78].

In a study conducted by Nishikawa et al., UPECs were injected transurethrally into the mouse bladder. Then, purified phages T4 and KEP10 (Myoviridae family) were immediately injected into the peritoneal cavity of mice to treat UTI. The findings manifested that phages could save a large percentage of mice (up to $90 \%$ ) from death through a dose-dependent infection. On the other hand, all mice in the control group that were not treated after infection died within 3 days. Notably, no specific side effects were reported for any of the phages. Moreover, the results illustrated that these phages moved rapidly into the bloodstream and disseminated into internal organs and they remained stable for $24 \mathrm{~h}$ at most in the human and mouse urine samples at $37^{\circ} \mathrm{C}$. Therefore, the authors reported T4 and KEP10 as suitable therapeutic phages for treating lethal UTI [79]. Finally, in another in vivo study on mice, UTI was induced by transurethral application of Cronobacter turicensis. At the same time, specific phages against this bacterium were administered intraperitoneally. The authors reported that phage therapy decreased the number of bacteria in the kidney by $70 \%$. Phage therapy reduced the higher levels of malondialdehyde by $39 \%$ without any effect on the antioxidant status. Notably, there was no significant difference in Cronobacter colonization of the bladder between infected and phage treated groups. Furthermore, the expression of pro-inflammatory cytokines such as monocyte chemoattractant protein-1 and tumor necrosis factor-alpha increased due to the infection, while it was attenuated by phage therapy. The authors report that differences in the function of phages in the kidney and bladder can be related to the kinetics of phages after intraperitoneal administration, which could be improved by choosing another application route [80].

Therefore, the finding of various studies represented that phage therapy could be used to control UTI caused by MDR bacteria, although there are still many limitations in this treatment regimen. The administration of phages varied in different studies and there is still no comprehensive guideline on how to use them to ensure the highest clinical performance. Therefore, more clinical trials are needed to fully evaluate the safety and efficiency of potential therapeutic applications in vivo. Further, when a specific phage is used alone, the risk of development of phage-resistant mutant is very high. In this regard, the use of phage cocktails can be an effective solution because different phages use different receptors to minimize the development of resistance and widen the host range, which is important in the case of narrow host range infecting phages. However, as reported in the mentioned studies, resistance to cocktails has also occurred, but their molecular mechanisms have not yet been elucidated. Thus, the combined use of phage cocktails and different antibiotics increases the chances for controlling the UTI. At last, those antibiotics that are effective against uropathogenic bacteria such as polymixin and tigecycline are not usually used for a long time due to their many side effects, especially nephrotoxicity, although various studies have not reported the specific side effects of phages, which could lead to their widespread use in treatment of UTIs.

\section{Conclusion}

MDR Uropathogenic bacteria have reduced the utility of chemical antibiotics in clinical settings. Moreover, these antibiotics are cytotoxic to not only pathogens, but also health-beneficial commensals. In this regard, phages and their derivatives were reported as an alternative strategy for the treatment of drug-resistant UTI. Recent studies found that phages, in addition to inhibiting uropathogenic bacteria, could destroy their biofilm. Besides, the safety profile of these microorganism seems to be far better than antibiotics. Therefore, researchers suggested phage therapy as a mean for prevention and treatment of UTI and further spread of MDR uropathogenic bacteria. However, phage-resistant strains still occur despite the use of phage cocktails and combination therapy. In this context, more fundamental studies are required to determine the phage-host interactions and the phage potential to control UTI. Additionally, most of phage therapy data were obtained from in vitro studies and the major limitation was lack of appropriate clinical research. So, 
further clinical trials (double-blind and placebo-control) are needed to investigate dose, best routes of administration, frequency and duration of phage therapy for inhibition and treatment of UTI.

\section{Acknowledgements}

We greatly appreciate the input from Melika Khanzadeh Tehrani (from Department of Pathobiology, School of Public Health, Tehran University of Medical Sciences, Tehran, Iran) for her collaboration with us in figure design.

\section{Authors' contributions}

AS conceived and designed the study. AS, AM and SV contributed in comprehensive research. AS, ZC and AK wrote the paper. AH participated in manuscript editing. Notably, all authors have read and approved the manuscript.

\section{Funding}

Not applicable.

\section{Availability of data and materials}

The authors confirm that the data supporting the findings of this study is available within the article

\section{Declarations}

\section{Ethics approval and consent to participate}

Not applicable.

\section{Consent for publication}

Not applicable.

\section{Competing interests}

The authors declare that they have no competing interests.

\begin{abstract}
Author details
'Department of Microbiology, School of Medicine, Iran University of Medical Sciences, Tehran, Iran. ${ }^{2}$ Department of Molecular Genetics, Faculty of Basic Sciences and Advanced Technologies in Biology, University of Science and Culture, Tehran, Iran. ${ }^{3}$ Department of Medical Laboratory Sciences, Khomein University of Medical Sciences, Khomein, Iran. ${ }^{4}$ Department of Microbiology, School of Medicine, Shahid Beheshti University of Medical Sciences, Tehran, Iran.
\end{abstract}

Received: 22 January 2021 Accepted: 13 April 2021

Published online: 26 April 2021

\section{References}

1. de Miguel T, Rama JLR, Sieiro C, Sánchez S, Villa TG. Bacteriophages and lysins as possible alternatives to treat antibiotic-resistant urinary tract infections. Antibiotics. 2020:9:466.

2. Malik S, Sidhu PK, Rana J, Nehra K. Managing urinary tract infections through phage therapy: a novel approach. Folia Microbiologica. 2020;65:217-31.

3. Zalewska-Piątek B, Piątek R. Phage therapy as a novel strategy in the treatment of urinary tract infections caused by E. coli. Antibiotics. 2020;9:304

4. Cassini A, Plachouras D, Eckmanns T, Abu Sin M, Blank H-P, Ducomble T, Haller S, Harder T, Klingeberg A, Sixtensson M. Burden of six healthcareassociated infections on European population health: estimating incidence-based disability-adjusted life years through a population prevalence-based modelling study. PLoS Med. 2016;13:e1002150.

5. Flores-Mireles AL, Walker JN, Caparon M, Hultgren SJ. Urinary tract infections: epidemiology, mechanisms of infection and treatment options. Nat Rev Microbiol. 2015;13:269-84.

6. Hotchandani R, Aggarwal K. Urinary tract infections in women. 2012.

7. Foxman B. The epidemiology of urinary tract infection. Nat Rev Urol. 2010;7:653-60

8. Stamm WE. Scientific and clinical challenges in the management of urinary tract infections. Am J Med. 2002:113:1-4.
9. Kline KA, Bowdish DM. Infection in an aging population. Curr Opin Microbiol. 2016;29:63-7.

10. Grygorcewicz B, Wojciuk B, Roszak M, Łubowska N, Błażejczak P, JursaKulesza J, Rakoczy R, Masiuk H, Dołęgowska B. Environmental phagebased cocktail and antibiotic combination effects on Acinetobacter baumannii biofilm in a human urine model. Microbial Drug Resist. 2020;27:25-35

11. Pires DP, Melo LD, Boas DV, Sillankorva S, Azeredo J. Phage therapy as an alternative or complementary strategy to prevent and control biofilm-related infections. Curr Opin Microbiol. 2017;39:48-56.

12. Chegini Z, Khoshbayan A, Moghadam MT, Farahani I, Jazireian P, Shariati A. Bacteriophage therapy against Pseudomonas aeruginosa biofilms: a review. Ann Clin Microbiol Antimicrob. 2020;19:1-17.

13. Moghadam MT, Amirmozafari N, Shariati A, Hallajzadeh M, Mirkalantari S, Khoshbayan A, Jazi FM. How phages overcome the challenges of drug resistant bacteria in clinical infections. Infection drug resistance. 2020;13:45

14. Lin DM, Koskella B, Lin HC. Phage therapy: an alternative to antibiotics in the age of multi-drug resistance. World J Gastrointest Pharmacol Ther. 2017:8:162-73.

15. Sabino J, Hirten RP, Colombel JF. Review article: bacteriophages in gastroenterology-from biology to clinical applications. Aliment Pharmacol Ther. 2020;51:53-63.

16. LU TK, Collins JJ. Dispersing biofilms with engineered enzymatic bacteriophage. Proc Natl Acad Sci. 2007;104:11197-202.

17. Łusiak-Szelachowska M, Weber-Dąbrowska B, Górski A. Bacteriophages and lysins in biofilm control. Virologica Sinica. 2020;35:1-9.

18. Jaiswal A, Koley H, Ghosh A, Palit A, Sarkar B. Efficacy of cocktail phage therapy in treating Vibrio cholerae infection in rabbit model. Microbes Infect. 2013;15:152-6.

19. Moghadam MT, Khoshbayan A, Chegini Z, Farahani I, Shariati A. Bacteriophages, a New Therapeutic Solution for Inhibiting Multidrug-Resistant Bacteria Causing Wound Infection: Lesson from Animal Models and Clinical Trials. Drug Design Development Therapy. 2020;14:1867.

20. Bao J, Wu N, Zeng Y, Chen L, Li L, Yang L, Zhang Y, Guo M, Li L, Li J. Nonactive antibiotic and bacteriophage synergism to successfully treat recurrent urinary tract infection caused by extensively drug-resistant Klebsiella pneumoniae. Emerging Microbes Infections. 2020;9:771-4.

21. Liao KS, Lehman SM, Tweardy DJ, Donlan RM, Trautner BW. Bacteriophages are synergistic with bacterial interference for the prevention of Pseudomonas aeruginosa biofilm formation on urinary catheters. J Appl Microbiol. 2012:113:1530-9.

22. Yoon SH, Jeong H, Kwon S-K, Kim JF. Genomics, biological features, and biotechnological applications of Escherichia coli B:"Is B for better?!". In Systems biology and biotechnology of Escherichia coli. Springer; 2009: $1-17$.

23. Allocati N, Masulli M, Alexeyev MF, Di llio C. Escherichia coli in Europe: an overview. Int J Environ Res Public Health. 2013;10:6235-54.

24. Kaper JB, Nataro JP, Mobley HL. Pathogenic escherichia coli. Nature reviews microbiology. 2004;2:123-40.

25. Ochoa SA, Cruz-Córdova A, Luna-Pineda VM, Reyes-Grajeda JP, CázaresDomínguez V, Escalona G, Sepúlveda-González M, López-Montiel F, Arellano-Galindo J, López-Martínez B: Multidrug-and extensively drug-resistant uropathogenic Escherichia coli clinical strains: phylogenetic groups widely associated with integrons maintain high genetic diversity. Front Microbiol 2016,7:2042.

26. Yazdi M, Bouzari M, Ghaemi EA, Shahin K. Isolation, characterization and genomic analysis of a novel bacteriophage VB_EcoS-Golestan infecting multidrug-resistant Escherichia coli isolated from urinary tract infection. Sci Rep. 2020;10:1-13.

27. Gu Y, Xu Y, Xu J, Yu X, Huang X, Liu G, Liu X. Identification of novel bacteriophage $v B$ ECCOP-EG1 with lytic activity against planktonic and biofilm forms of uropathogenic Escherichia coli. Appl Microbiol Biotechnol. 2019:103:315-26.

28. Chibeu A, Lingohr EJ, Masson L, Manges A, Harel J, Ackermann H-W, Kropinski AM, Boerlin P. Bacteriophages with the ability to degrade uropathogenic Escherichia coli biofilms. Viruses. 2012;4:471-87.

29. Valério N, Oliveira $C$, Jesus V, Branco T, Pereira C, Moreirinha C, Almeida A. Effects of single and combined use of bacteriophages and antibiotics to inactivate Escherichia coli. Virus Res. 2017;240:8-17. 
30. Moradpour Z, Yousefi N, Sadeghi D, Ghasemian A. Synergistic bactericidal activity of a naturally isolated phage and ampicillin against urinary tract infecting Escherichia coli O157. Iran J Basic Med Sci. 2020;23:257.

31. Comeau AM, Tétart F, Trojet SN, Prere M-F, Krisch H. Phage-antibiotic synergy (PAS): $\beta$-lactam and quinolone antibiotics stimulate virulent phage growth. Plos ONE. 2007;2:e799.

32. Chan BK, Sistrom M, Wertz JE, Kortright KE, Narayan D, Turner PE. Phage selection restores antibiotic sensitivity in MDR Pseudomonas aeruginosa Sci Rep. 2016;6:26717.

33. Wishart DS, Knox C, Guo AC, Shrivastava S, Hassanali M, Stothard P, Chang Z, Woolsey J. DrugBank: a comprehensive resource for in silico drug discovery and exploration. Nucleic Acids Res. 2006;34:D668-72.

34. Rózalski A, Sidorczyk Z, Kotełko K. Potential virulence factors of Proteus bacilli. Microbiol Mol Biol Rev. 1997;61:65-89.

35. Rubin R, Tolkoff-Rubin N, Cotran R. Urinary tract infection, pyelonephritis, and reflux nephropathy. The kidney. 1986;2:1085-141.

36. Warren J. Clinical presentations and epidemiology of urinary tract infections. Urinary tract infections: molecular pathogenesis and clinical management. Washington: ASM Press; 1996. p. 3-27.

37. Armbruster CE, Smith SN, Johnson AO, DeOrnellas V, Eaton KA, Yep A Mody L, Wu W, Mobley HL. The pathogenic potential of Proteus mirabilis is enhanced by other uropathogens during polymicrobial urinary tract infection. Infect Immun. 2017;85:e00808.

38. Hooton TM, Bradley SF, Cardenas DD, Colgan R, Geerlings SE, Rice JC, Saint S, Schaeffer AJ, Tambayh PA, Tenke P. Diagnosis, prevention, and treatment of catheter-associated urinary tract infection in adults: 2009 International Clinical Practice Guidelines from the Infectious Diseases Society of America. Clin Infect Dis. 2010;50:625-63.

39. Jacobsen S, Stickler D, Mobley H, Shirtliff M. Complicated catheter-associated urinary tract infections due to Escherichia coli and Proteus mirabilis. Clin Microbiol Rev. 2008:21:26-59.

40. Wang J-T, Chen P-C, Chang S-C, Shiau Y-R, Wang H-Y, Lai J-F, Huang I-W, Tan M-C, Lauderdale T-LY. Antimicrobial susceptibilities of Proteus mirabilis: a longitudinal nationwide study from the Taiwan surveillance of antimicrobial resistance (TSAR) program. BMC Infect Dis. 2014;14:486.

41. Viertel TM, Ritter K, Horz H-P. Viruses versus bacteria-novel approaches to phage therapy as a tool against multidrug-resistant pathogens. J Antimicrob Chemother. 2014;69:2326-36.

42. Melo LD, Veiga P, Cerca N, Kropinski AM, Almeida C, Azeredo J, Sillankorva S. Development of a phage cocktail to control Proteus mirabilis catheterassociated urinary tract infections. Front Microbiol. 2016;7:1024.

43. Maszewska A, Zygmunt M, Grzejdziak I, Różalski A. Use of polyvalent bacteriophages to combat biofilm of Proteus mirabilis causing catheterassociated urinary tract infections. J Appl Microbiol. 2018;125:1253-65.

44. Tait K, Skillman L, Sutherland IW. The efficacy of bacteriophage as a method of biofilm eradication. Biofouling. 2002;18:305-11.

45. Sutherland IW, Hughes KA, Skillman LC, Tait K. The interaction of phage and biofilms. FEMS Microbiol Lett. 2004;232:1-6.

46. Nzakizwanayo J, Hanin A, Alves DR, McCutcheon B, Dedi C, Salvage J, Knox K, Stewart B, Metcalfe A, Clark J. Bacteriophage can prevent encrustation and blockage of urinary catheters by Proteus mirabilis. Antimicrob Agents Chemother. 2016;60:1530-6.

47. Fu W, Forster T, Mayer O, Curtin JJ, Lehman SM, Donlan RM. Bacteriophage cocktail for the prevention of biofilm formation by Pseudomonas aeruginosa on catheters in an in vitro model system. Antimicrob Agents Chemother. 2010;54:397-404

48. Gomaa S, Serry F, Abdellatif H, Abbas H. Elimination of multidrugresistant Proteus mirabilis biofilms using bacteriophages. Arch Virol. 2019;164:2265-75.

49. Yazdi M, Bouzari M, Ghaemi EA. Isolation and characterization of a lytic bacteriophage (VB_PmiS-TH) and its application in combination with ampicillin against planktonic and biofilm forms of Proteus mirabilis isolated from urinary tract infection. J Mol Microbiol Biotechnol. 2018;28:37-46.

50. Broomfield RJ, Morgan SD, Khan A, Stickler DJ. Crystalline bacterial biofilm formation on urinary catheters by urease-producing urinary tract pathogens: a simple method of control. J Med Microbiol. 2009;58:1367-75.

51. Wasfi R, Hamed SM, Amer MA, Fahmy LI. Proteus mirabilis biofilm: development and therapeutic strategies. Front Cell Infect Microbiol. 2020:10:414-4.
52. Bahramian A, Shariati A, Azimi T, Sharahi JY, Bostanghadiri N, Gachkar L, Ghalavand Z, Chirani AS, Erfanimanesh S, Hashemi A. First report of New Delhi metallo- $\beta$-lactamase-6 (NDM-6) among Klebsiella pneumoniae ST147 strains isolated from dialysis patients in Iran. Infect Genet Evol. 2019;69:142-5.

53. Tabassum R, Shafique M, Khawaja KA, Alvi IA, Rehman Y, Sheik CS, Abbas $Z$, ur Rehman S. Complete genome analysis of a Siphoviridae phage TSK1 showing biofilm removal potential against Klebsiella pneumoniae. Sci Rep. 2018:8:1-11.

54. Moghadam M, Shariati A, Mirkalantari S, Karmostaji A. The complex genetic region conferring transferable antibiotic resistance in multidrugresistant and extremely drug-resistant Klebsiella pneumoniae clinical isolates. New Microb New Infections 2020, 36:100693

55. Horváth M, Kovács T, Koderivalappil S, Ábrahám H, Rákhely G, Schneider $\mathrm{G}$. Identification of a newly isolated lytic bacteriophage against K24 capsular type, carbapenem resistant Klebsiella pneumoniae isolates. Sci Rep. 2020;10:1-11.

56. Sybesma W, Zbinden R, Chanishvili N, Kutateladze M, Chkhotua A, Ujmajuridze A, Mehnert U, Kessler TM. Bacteriophages as potential treatment for urinary tract infections. Front Microbiol. 2016;7:465.

57. Verma V, Harjai K, Chhibber S. Characterization of a T7-like lytic bacteriophage of Klebsiella pneumoniae B5055: a potential therapeutic agent. Current microbiology. 2009;59:274-81.

58. Zhang R, Zhao F, Wang J, Pei G, Fan H, Zhangxiang L, Mi Z, Shi T, Liu H, Tong Y. Biological characteristics and genome analysis of a novel phage vB_KpnP_IME279 infecting Klebsiella pneumoniae. Folia Microbiologica 2020;65:1-12.

59. Ko KS. The contribution of capsule polysaccharide genes to virulence of Klebsiella pneumoniae. Virulence. 2017;8:485.

60. Tan D, Zhang Y, Cheng M, Le S, Gu J, Bao J, Qin J, Guo X, Zhu T. Characterization of Klebsiella pneumoniae ST11 isolates and their interactions with lytic phages. Viruses. 2019;11:1080.

61. Hsu C-R, Lin T-L, Pan Y-J, Hsieh P-F, Wang J-T. Isolation of a bacteriophage specific for a new capsular type of Klebsiella pneumoniae and characterization of its polysaccharide depolymerase. PloS ONE. 2013;8:e70092.

62. Bernasconi $O J$, Donà $V$, Tinguely R, Endimiani $A$. In vitro activity of three commercial bacteriophage cocktails against multidrug-resistant Escherichia coli and Proteus spp. strains of human and non-human origin. J Glob Antimicrob Resist. 2017;8:179-85.

63. Pirnay J-P, Blasdel BG, Bretaudeau L, Buckling A, Chanishvili N, Clark JR, Corte-Real S, Debarbieux L, Dublanchet A, De Vos D. Quality and safety requirements for sustainable phage therapy products. Pharm Res. 2015;32:2173-9.

64. Vandenheuvel D, Lavigne R, Brüssow H. Bacteriophage therapy: advances in formulation strategies and human clinical trials. Annu Rev Virol. 2015:2:599-618.

65. Pereira S, Pereira C, Santos L, Klumpp J, Almeida A. Potential of phage cocktails in the inactivation of Enterobacter cloacae-An in vitro study in a buffer solution and in urine samples. Virus Res. 2016;211:199-208.

66. Rigvava S, Tchgkonia I, Jgenti D, Dvalidze T, Carpino J, Goderdzishvili M. Comparative analysis of the biological and physical properties of Enterococcus faecalis bacteriophage vB_EfaS_GEC-EfS_3 and Streptococcus mitis bacteriophage vB_SmM_GEC-SmitisM_2. Can J Microbiol. 2013;59:18-21.

67. Han SH, Lee D, Im J, Na H, Ryu S, Yun C-H. The novel Enterococcus phage VB_EfaS_HEf13 has broad lytic activity against clinical isolates of Enterococcus faecalis. Front Microbiol. 2019;10:2877.

68. Yazdi M, Bouzari M, Ghaemi EA. Isolation and characterization of a potentially novel Siphoviridae phage (vB_SsapS-104) with lytic activity against Staphylococcus saprophyticus isolated from urinary tract infection. Folia microbiologica. 2019;64:283-94.

69. Tan CAZ, Antypas H, Kline KA. Overcoming the challenge of establishing biofilms in vivo: a roadmap for Enterococci. Curr Opin Microbiol. 2020;53:9-18.

70. Saggu SK, Jha G, Mishra PC. Enzymatic degradation of biofilm by metalloprotease from Microbacterium sp. SKS10. Front Bioeng Biotechnol. 2019;7:192.

71. Yang D, Chen $Y$, Sun E, Hua L, Peng Z, Wu B. Characterization of a lytic bacteriophage vB_EfaS_PHB08 harboring endolysin Lys08 against Enterococcus faecalis biofilms. Microorganisms. 2020;8:1332. 
72. Lehman SM, Donlan RM. Bacteriophage-mediated control of a twospecies biofilm formed by microorganisms causing catheter-associated urinary tract infections in an in vitro urinary catheter model. Antimicrob Agents Chemother. 2015;59:1127-37.

73. Kay MK, Erwin TC, McLean RJ, Aron GM. Bacteriophage ecology in Escherichia coli and Pseudomonas aeruginosa mixed-biofilm communities. Appl Environ Microbiol. 2011;77:821-9.

74. Macleod SM, Stickler DJ. Species interactions in mixed-community crystalline biofilms on urinary catheters. J Med Microbiol. 2007:56:1549-57.

75. Rostkowska OM, Międzybrodzki R, Miszewska-Szyszkowska D, Górski A, Durlik M. Treatment of recurrent urinary tract infections in a 60-year-old kidney transplant recipient. The use of phage therapy. Transplant Infectious Disease 2020;23:e13391.

76. Schooley RT, Biswas B, Gill JJ, Hernandez-Morales A, Lancaster J, Lessor L, Barr JJ, Reed SL, Rohwer F, Benler S. Development and use of personalized bacteriophage-based therapeutic cocktails to treat a patient with a disseminated resistant Acinetobacter baumannii infection. Antimicrobial agents and chemotherapy 2017,61.

77. Roach DR, Leung CY, Henry M, Morello E, Singh D, Di Santo JP, Weitz JS, Debarbieux L. Synergy between the host immune system and bacteriophage is essential for successful phage therapy against an acute respiratory pathogen. Cell Host Microbe. 2017;22:38-47. e34.

78. Ujmajuridze A, Chanishvili N, Goderdzishvili M, Leitner L, Mehnert U, Chkhotua A, Kessler TM, Sybesma W. Adapted bacteriophages for treating urinary tract infections. Front Microbiol. 2018;9:1832.

79. Nishikawa H, Yasuda M, Uchiyama J, Rashel M, Maeda Y, Takemura I, Sugihara S, Ujihara T, Shimizu Y, Shuin T. T-even-related bacteriophages as candidates for treatment of Escherichia coli urinary tract infections. Arch Virol. 2008;153:507-15.

80. Tóthová L’, Celec P, Bábíčková J, Gajdošová J, Al-Alami H, Kamodyova N, Drahovska H, Liptakova A, Turňa J, Hodosy J. Phage therapy of Cronobacter-induced urinary tract infection in mice. Med Sci Monit. 2011;17:BR173.

\section{Publisher's note}

Springer Nature remains neutral with regard to jurisdictional claims in published maps and institutional affiliations.
Ready to submit your research? Choose BMC and benefit from:

- fast, convenient online submission

- thorough peer review by experienced researchers in your field

- rapid publication on acceptance

- support for research data, including large and complex data types

- gold Open Access which fosters wider collaboration and increased citations

- maximum visibility for your research: over $100 \mathrm{M}$ website views per year

At BMC, research is always in progress.

Learn more biomedcentral.com/submissions 